\title{
Efficacy and Mechanism of Ultrasound Combined with Slightly Acidic Electrolyzed Water for Inactivating Escherichia coli
}

\author{
Liping Guo, ${ }^{1}$ Xuecong Zhang, ${ }^{1}$ Lin Xu, ${ }^{1}$ Yan $\mathrm{Li},{ }^{1}$ Bin Pang, ${ }^{1}$ Jingxin Sun $\mathbb{D},{ }^{1,2}$ \\ Baowei Wang, ${ }^{1,2}$ Ming Huang, ${ }^{3}$ Xinglian $\mathrm{Xu}^{3}$ and Harvey $\mathrm{Ho}^{4}$ \\ ${ }^{1}$ College of Food Science and Engineering, Shandong Reseach Center for Meat Food Quality Control, \\ Qingdao Agricultural University, Qingdao 266109, China \\ ${ }^{2}$ Qingdao Special Food Research Institute, Qingdao 266109, China \\ ${ }^{3}$ Key Laboratory of Meat Processing \& Quality Control, College of Food Science \& Technology, Nanjing Agricultural University, \\ Nanjing 210095, China \\ ${ }^{4}$ Auckland Bioengineering Institute, The University of Auckland, Auckland, New Zealand
}

Correspondence should be addressed to Jingxin Sun; jxsunqau@126.com

Received 17 December 2020; Revised 17 February 2021; Accepted 1 March 2021; Published 9 March 2021

Academic Editor: Qing-Jie Sun

Copyright (c) 2021 Liping Guo et al. This is an open access article distributed under the Creative Commons Attribution License, which permits unrestricted use, distribution, and reproduction in any medium, provided the original work is properly cited.

In the present study, the synergetic effect and mechanism of ultrasound (US) and slightly acidic electrolyzed water (SAEW) on the inactivation of Escherichia coli (E. coli) were evaluated. The results showed that US combined with SAEW treatment showed higher sanitizing efficacy for reducing $E$. coli than US and SAEW alone treatment. US and US combined with SAEW treatments resulted in smaller particle size of E. coli compared to the control and SAEW treatment. In addition, US combined with SAEW treatment induced the highest potassium leakage. However, the highest protein leakage was recorded in US treatment. Moreover, scanning and transmission electron microscopy analysis revealed that the greatest damage of the appearance and ultrastructure of E. coli was achieved after US combined with SAEW treatment. The synergetic effect was also confirmed by CLSM analysis. Fluorescence spectroscopy suggested that treatments of US, SAEW, and US combined with SAEW changed protein conformation of E. coli. Overall, the present study demonstrated that the sterilization mechanism of US combined with SAEW treatment was decreasing the particle size and disrupting the permeability of cell membrane and the cytoplasmic ultrastructure as well as changing protein conformation of $E$. coli.

\section{Introduction}

Escherichia coli (E. coli), a Gram-negative rod-shaped bacterium, is widely recognized as one of the major food-borne pathogens often from the consumption of contaminated foods $[1,2]$. Therefore, a great variety of sanitization strategies, including heating, ultrasound, ultraviolet-C, sodium hypochlorite, sodium benzoate, potassium sorbate, chlorine dioxide, and so on, have been used to reduce the microbial population and prolong the shelf life of food products by the food industry [3-5]. Even so, a more effective and much safer sterilizing technology is crucial for environmental conservation and food preservation.
Ultrasound, as a promising non-thermal sterilization technique, can cause physical effects and/or chemical effects, thus decontaminating microorganisms from the surfaces of foods. Koda et al. [6] reported that inactivation of microorganisms by high frequency ultrasound was mainly dependent on the chemical effects. On the other hand, reactive oxygen species generated by cavitation also assisted sterilization during ultrasound processing [7]. In recent years, many literatures reported that sterilization effect of combined treatment of ultrasound with chemical sterilant was more effective than each used alone [8]. Our results showed that ultrasound produced micro-cracks in the bacterial cell membranes, allowing $\mathrm{NaOCl}$ into the cells, and thus 
deactivated $E$. coli and reduced the usage amount of sodium hypochlorite [9]. In China, non- thermal sterilization has not been widely used in food industry. $\mathrm{NaOCl}$ also has been widely used in sterilization in our country, but its security is a concern.

The slightly acidic electrolyzed water (SAEW), as an alternative and novel method with great potential for sterilization, has recently received a great deal of attention for its sanitizing efficacy and environmentally friendly nature [10-13]. Hypochlorous acid ( $\mathrm{HClO})$ produced by SAEW can inactivate the microbial cell via improving the oxidation of lipids and protein compounds as well as resulting in a modification in the electron transfer mechanism of microorganisms [14]. The results of Naka et al. [12] indicated that, at the same chlorine concentration, SAEW is more effective than $\mathrm{NaOCl}$ in reducing or eliminating bacterial count. Recently, some studies investigated the synergistic action of electrolyzed water and ultrasound on microbial inactivation. José Cichoski et al. [14] reported that the combination of US and SAEW could effectively reduce some microorganisms, including enterobacteria, mesophilic bacteria, lactic acid bacteria, and psychrotrophic bacteria, and thus has a great potential to improve the prechilling of poultry carcasses. In addition, SAEW simultaneous with US treatment at $40^{\circ} \mathrm{C}$ for $3 \mathrm{~min}$ showed the synergic effects against $B$. cereus on potato [10]. Scanning and transmission electron microscopy analysis revealed that combined ultrasound-SAEW treatment resulted in greater damage of Staphylococcus aureus than either treatment alone [15]. However, little information is available on the effect of US combined with SAEW against Escherichia coli and the related mechanism.

Therefore, in this study, the effects of US combined with SAEW treatment on the antibacterial activity, membrane permeability, membrane integrity, cell morphology, intracellular organization, and protein conformation of $E$. coli were investigated to the antibacterial mechanism of US combined with SAEW.

\section{Materials and Methods}

2.1. Microbial Inoculation. Escherichia coli CICC 10899 was obtained from Chinese Center of Industrial Culture Collection). The stock cultures were transferred to $50 \mathrm{~mL}$ of nutrient broth (NB) (Hiabo Bio-Tech Co., Qingdao, China) and incubated at $37{ }^{\circ} \mathrm{C}$ in an air bath incubator with a reciprocal shaker for $16 \mathrm{~h}$ at $150 \mathrm{rpm}$. Following incubation, the microbial culture was sedimented by centrifugation at $6,000 \times \mathrm{g}$ for $10 \mathrm{~min}$ at $4^{\circ} \mathrm{C}$. The supernatant was discarded and the bacterial cells were washed twice with $0.90 \%$ sterile saline solution and resuspended for following use. The final population in bacterial suspension of $E$. coli was approximately $10^{6} \mathrm{CFU} / \mathrm{mL}$.

2.2. SAEW Preparation. SAEW was produced by electrolysis with a continuous supply of dilute $\mathrm{NaCl}$ solution $(0.9 \%)$ in a chamber without a membrane using an electrolysis device (Anywhere-320W, Rui Andre Environmental Equipment
Co., Ltd., Beijing, China). SAEW pH and ORP values were determined immediately before sample treatment using a pH meter (Starter 300, Ohaus Co., USA) with $\mathrm{pH}$ and ORP electrodes. A colorimetric method with a digital chlorine test kit (RC-3F; Kasahara Chemical Instruments Corp., Saitama, Japan) was used to measure ACC. In this study, SAEW with a $\mathrm{pH}$ of 6.18 , ORP of $827 \mathrm{mV}$, and available chlorine concentration (ACC) of $30 \mathrm{mg} / \mathrm{L}$ was used to sterilize.

\subsection{Single or Combined Treatments with US and SAEW.} US treatment was applied using a probe-style ultrasonic processor (Scientz-II D; Ningbo Scientz, Zhejiang, China). A total of $27 \mathrm{~mL}$ of cell suspension was added with $3 \mathrm{~mL} 0.90 \%$ sterile saline solution, and then the ultrasonic emitter was immersed $2.0 \mathrm{~cm}$ into the solution and ultrasonically treated for $10 \mathrm{~min}$ at a frequency of $20 \mathrm{kHz}$ and $10 \mathrm{~W} / \mathrm{cm}^{3}$ energy density.

For SAEW treatment, the inoculated samples of $27 \mathrm{~mL}$ were mixed with $3 \mathrm{~mL}$ SAEW in a sterile glass beaker for $10 \mathrm{~min}$. For combined treatment, after mixing $3 \mathrm{~mL}$ SAEW in $27 \mathrm{~mL}$ cell suspension, the US treatment followed immediately for $10 \mathrm{~min}$ under the above ultrasonic conditions. In this study, a thermostatic water bath (DC-1006, Safe Corporation, Ningbo, China) was used to maintain the temperature at $20^{\circ} \mathrm{C}$ in order to prevent a lethal thermal effect after US treatment.

2.4. Microbiological Analysis. Following treatments, microbiological analysis was conducted using plate counting method according to the previous procedure [9]. After incubation, microbial colonies were counted with an automated plate counter (ProtoCOL, Synoptics, Cambridge, UK). All analyses were conducted in duplicate with 3 replicates for each experiment.

2.5. Microbiological Size Measurement. The Malvern Mastersizer 2000 (Malvern Instruments, UK) was used to measure the particle size measurement of bacterial suspensions according to the method described by Gao et al. [16].

2.6. Measurement of the Intracellular Protein Leakage and Potassium Leakage. After treatments, the suspension was centrifuged at $10000 \mathrm{~g}$ for $10 \mathrm{~min}$ at $4^{\circ} \mathrm{C}$. The protein content in the supernatant was according to the method of Bradford [17], using bovine serum albumin as standard.

The intracellular potassium leakage of the supernatant was determined using flame atomic absorption spectrophotometry (AAS) (AAnalyst 100, PerkinElmer Co., USA) as previously described by Tang et al. [18]. A linear relationship between potassium concentration and emission was obtained using potassium standards (analytical grade, Sigma-Aldrich, Poole, United Kingdom). The content of potassium was measured by AAS and calculated by the calibration. 
2.7. Scanning and Transmission Electron Microscopy Analysis. Scanning electron microscopy (SEM) was used to observe the morphological changes in E. coli cells according to the method of Li et al. [15]. After centrifugation at $10000 \mathrm{~g}$ for $10 \mathrm{~min}$ at $4^{\circ} \mathrm{C}$, the precipitates were collected and rinsed twice with $0.85 \%$ sterile saline solution. The samples were fixed with $2.5 \%$ glutaraldehyde for $24 \mathrm{~h}$ and then were washed three times with phosphate buffer solution ( $\mathrm{pH}$ 7.2) and post-fixed with $1 \%$ osmium tetroxide for $2 \mathrm{~h}$. Afterwards, the samples were dehydrated using a graded ethanol $(30,50,70,80,90,95$, and $100 \%)$ series and transferred to a mixture of ethanol and tertiary butanol $(v: v=1: 1)$ for approximately $30 \mathrm{~min}$. They were then placed in pure tertiary butanol. Finally, the dehydrated samples were coated with gold-palladium and observed using a JSM-7500F scanning electron microscope (JEOL Ltd., Tokyo, Japan).

For TEM analysis, the cells were infiltrated and embedded in Epon-812 after washing and dehydration. The prepared specimens were sliced to thin sections $70 \mathrm{~nm}$ and stained with uranyl acetate and alkaline lead citrate for 10 min. A HT7700 transmission electron microscope (Hitachi Ltd., Tokyo, Japan) was used to examine at $80 \mathrm{kV}$.

2.8. Confocal Laser Scanning Microscopy (CLSM) Analysis. To assess the damage to $E$. coli cell membranes following treatments with single and combination of US and SAEW, CLSM analysis was performed using the method of Kang et al. [7], with some modifications. Cell suspensions were incubated with dye buffer (LIVE/DEAD ${ }^{\circledR}$ BacLight Bacterial Viability Kits, L7012, ThermoFisher) and stained with $20 \mu \mathrm{M}$ propidium iodide (PI) in the dark for $30 \mathrm{~min}$ at room temperature. The mixture was washed with $1 \mathrm{~mL}$ sterile HEPES buffer ( $\mathrm{pH}$ 7.0) and then observed in a fluorescence microscope (TCS SP5, Leica, Germany).

2.9. Fluorescence Spectroscopy Experiments. All recordings of fluorescence, synchronous, and resonance light scattering spectra were carried out on a FL2700 luminescence spectrometer (Hitachi High-Technologies Corporation, Japan) with a quartz cell of $10 \mathrm{~mm}$ path length. The excitation and emission wavelength, excitation and emission bandwidths intervals, and scanning wavelength range were in accordance with our previous study [9].

2.10. Statistical Analyses. All experiments were performed in triplicate. Data were expressed as the mean \pm standard deviation (SD). Significant differences were determined using one-way analysis of variance (ANOVA) and Duncan's multiple range tests (SPSS 19.0, SPSS Inc., Chicago, IL, USA) at $p<0.05$.

\section{Results and Discussion}

3.1. Effect of US Combined with SAEW Treatment on the Microbicidal Efficiency and Particle Size Distribution. Microbial reduction values resulting from different treatments are shown in Figure 1(a). US treatment for $10 \mathrm{~min}$ decreased the number of $E$. coli by $0.48 \log \mathrm{CFU} / \mathrm{mL}$, indicating that the US treatment alone was not effective for inactivating E. coli CICC 10899. The similar phenomenon was also found in decontamination of $S$. aureus [15], E. coli ATCC 10536, and V. Parahaemolyticus KCTC 2471 [19]. The action of the US and SAEW is related to the species of bacteria. Park et al. [19] reported that SAEW treatment (chlorine $30 \mathrm{mg} / \mathrm{L}$ ) showed the higher sterilizing effect for $V$. Parahaemolyticus KCTC but showed the lower sterilizing effect for E. coli ATCC 10536 than US treatment for $50 \mathrm{~min}$. SAEW treatment also was not efficient in reducing Staphylococcus spp. [14]. However, in the present study, SAEW treatment led to 7.01-fold reduction of E. coli CICC 10899 when compared with US treatment for $10 \mathrm{~min}$. This result indicated that SAEW was an effective disinfectant for inactivating E. coli CICC 10899. The different phenomena of US and SAEW treatments on E. coli ATCC 10536 and E. coli CICC 10899 might have resulted from the difference of ultrasonic time. It also needs further confirmation.

Cichoski et al. [14] reported that SAEW combined with the application of US at $25 \mathrm{kHz}$ showed the synergistic effect on inactivating of enterobacteria, mesophilic bacteria, lactic acid bacteria, and psychrotrophic bacteria; however, SAEW combined with the application of US at $130 \mathrm{kHz}$ had no synergistic effect on inactivating of all bacteria. Moreover, for Staphylococcus spp., SAEW combined with US treatment did not increase and even decreased the inactivation efficacy compared to single US and SAEW treatment [14]. These results suggested that the synergistic effect of US combined with SAEW was also related to ultrasonic frequency and microbial species. In addition, SAEW combined with US treatment also significantly improved the reductions in the populations of inoculated S. aureus, B. cereus, E. coli O157: $\mathrm{H} 7$, and A. fumigatus in kashk compared to SAEW alone [20]. In the present study, US and SAEW treatment exhibited the synergistic effect in sterilization of E. coli and presented the highest reduction of $E$. coli with value of 3.64 $\log \mathrm{CFU} / \mathrm{mL}$. The reason might be that cavitation resulted from US disrupted cell membrane, accelerating SAEW into microbial cells, and thus inactivated E. coli CICC 10899.

Monomodal was observed in control and SAEW treated samples (Figure 1(b)). However, a small volume distribution at $100-500 \mathrm{~nm}$ was found after US and US + SAEW treatments. The average Sauter diameters of control and SAEW treated samples were $1688 \mathrm{~nm}$ and $1436 \mathrm{~nm}$, respectively. US and US + SAEW treatments caused significant reduction in particle size of $E$. coli, which was $718 \mathrm{~nm}$ and $762 \mathrm{~nm}$, respectively. These results indicated that the small distribution and decrease in particle size of E. coli were mainly attributed to cavitation of ultrasound rather than SAEW. The similar phenomenon was also observed by combined treatment of US and $\mathrm{NaOCl}[9]$.

3.2. Effect of US Combined with SAEW Treatment on the Intracellular Protein and Potassium Leakage. The protein and potassium leakage can be used to investigate the damage of the cell membranes [21, 22]. As shown in Figure 2, all treatments led to the leakage of intracellular protein and 


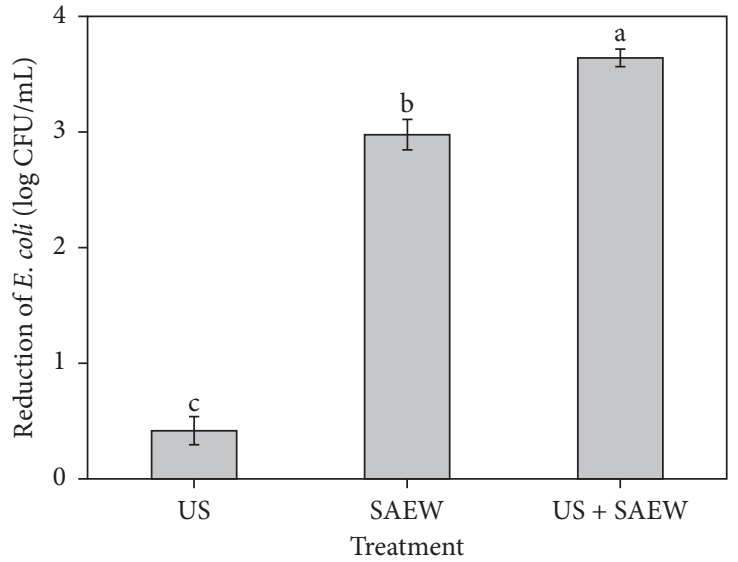

(a)

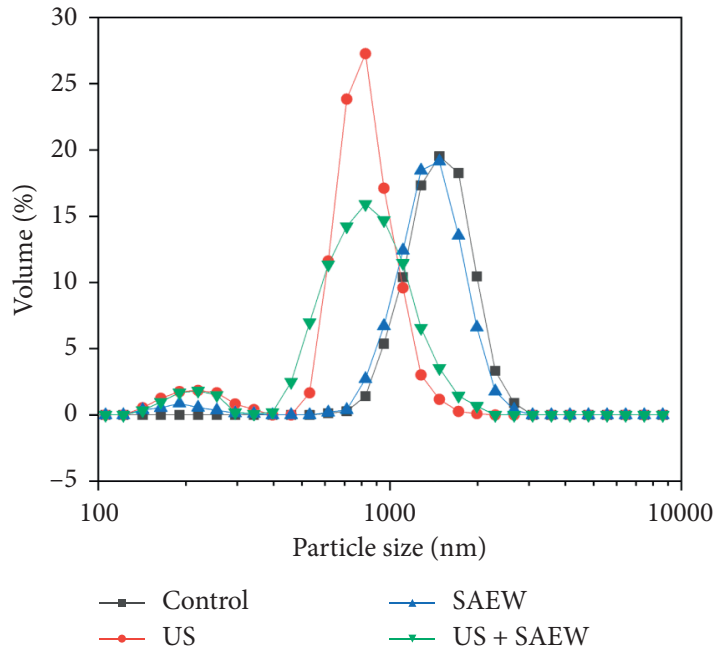

(b)

FiguRE 1: Reduction of E. coli (a) and particle size distribution (b) after the single and combined treatment of ultrasound and slightly acidic electrolyzed water. Values not sharing the same letter are significantly different at $p<0.05$. US: ultrasound, SAEW: slightly acidic electrolyzed water, and US + SAEW: ultrasound combined with slightly acidic electrolyzed water.

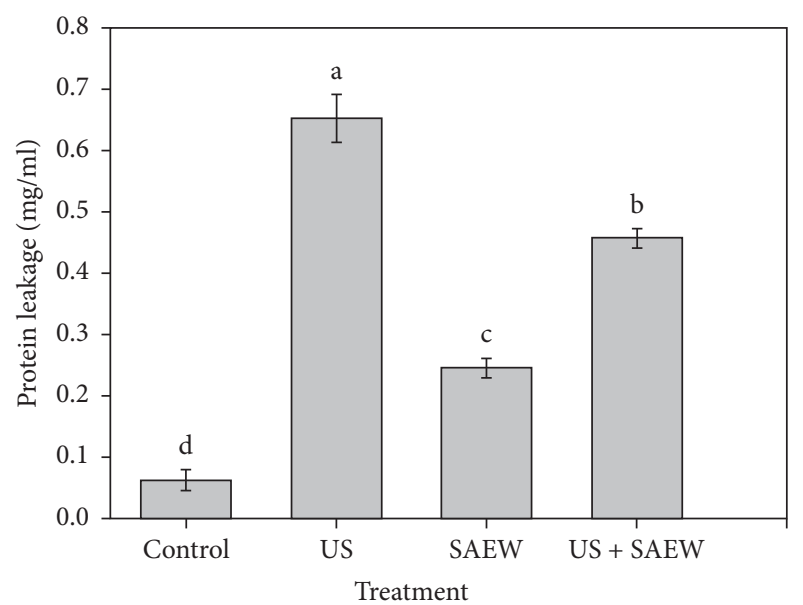

(a)

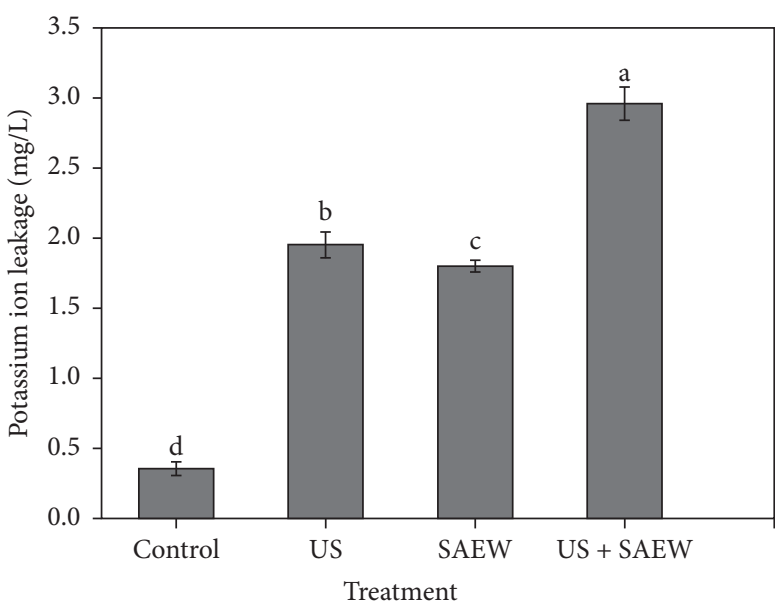

(b)

Figure 2: Protein leakage (a) and potassium ion leakage (b) of E. coli after the single and combined treatment of ultrasound and slightly acidic electrolyzed water. Values not sharing the same letter are significantly different at $p<0.05$. US: ultrasound, SAEW: slightly acidic electrolyzed water, and US + SAEW: ultrasound combined with slightly acidic electrolyzed water.

potassium of E. coli. After US, SAEW, and US + SAEW treatments, the protein concentrations in suspension increased to $0.65,0.25$, and $0.46 \mathrm{mg} / \mathrm{mL}$, respectively. On the other hand, US + SAEW treatment caused the highest potassium leakage, which was increased by $44.2 \%$ and $64.3 \%$ compared to US and SAEW treatment, respectively, indicating that US + SAEW treatment led to the most serious damage of the cell membranes of E. coli.

\subsection{Morphological Changes Revealed by Electron Microscopy.} Morphological changes of $E$. coli induced by US and SAEW were observed using SEM and TEM. SEM micrographs revealed that the cells of control samples maintained intact shapes, but with markedly deformation after SAEW treatment. E. coli was found markedly shrunk and cell wall was collapsed (Figure 3). This phenomenon could be due to the oxidative damage and the permeability of the cell membrane, thus resulting in the leakage of intracellular protein and potassium, and throwing the osmotic pressure out of balance $[15,23]$. While US and US + SAEW treatments resulted in more serious damage compared to SAEW treatment, in addition to shrink and collapse, cell membrane and cell wall of $E$. coli were also damaged.

The TEM micrographs of $E$. coli after treatments with US, SAEW, and US + SAEW are shown in Figure 4. For control samples, cell wall and membrane of $E$. coli. were continuous and intact and well defined. SAEW treatment 


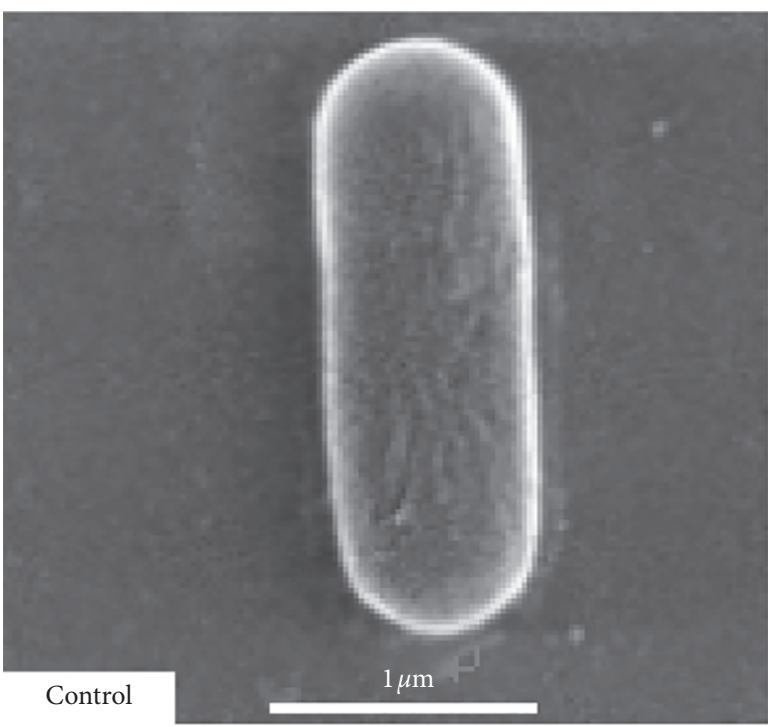

(a)

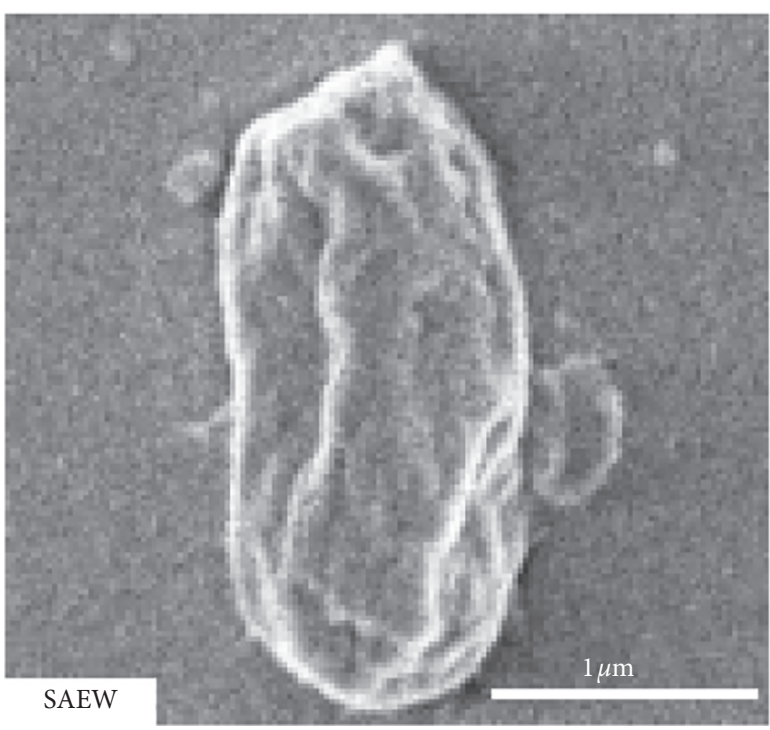

(c)

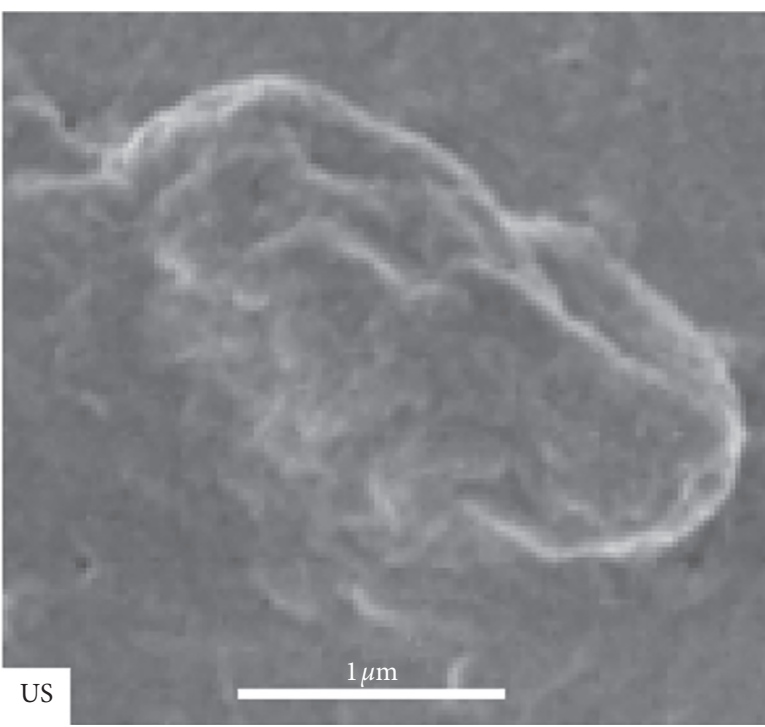

(b)

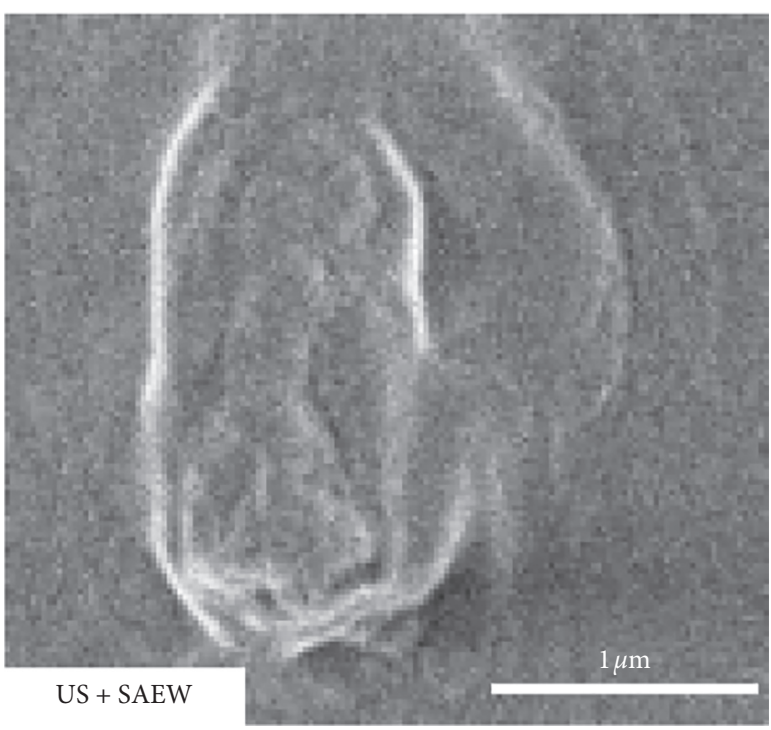

(d)

Figure 3: Scanning electron microscopy of E. coli after the single and combined treatment of ultrasound and slightly acidic electrolyzed water. Images were taken at magnification of $\times 20 \mathrm{~K}$. US: ultrasound, SAEW: slightly acidic electrolyzed water, and US + SAEW: ultrasound combined with slightly acidic electrolyzed water.

resulted in slight damage of cell wall. However, after US treatment, cell wall and membrane of E. coli were damaged and indefinite. US + SAEW treatment led to the most serious damage; meanwhile, a plenty of intracellular compounds also leaked. US could rupture the chemical bonds between molecular components in cell membranes, thus accelerating SAEW into bacteria $[15,24]$. Hence, the release of cell contents and disintegration of the cell wall was mainly attributed to the action of ultrasound on the damaged cells.

\subsection{CLSM Analysis of E. coli Under US Combined with SAEW} Treatment. The cells of $E$. coli with intact cell membranes were stained with fluorescent green, whereas cells with a damaged membrane were stained by red PI [25]. Figure 5 shows the live and dead population of $E$. coli after different treatments. A small fraction of dead cells was found after US and SAEW alone treatment. However, after treatment of US combined with SAEW, nearly all cells of E. coli showed red, indicating cytoplasmic membrane of most treated cells was injured, which was in accordance with results of microbial reduction values, SEM, and TEM analysis (Figures 1, 3, and 4).

3.5. The Effect of US Combined with SAEW Treatment on the Membrane Protein of E. coli. The conformational changes of proteins of $E$. coli can be successfully investigated by fluorescence spectroscopy [26, 27], since the intrinsic fluorescence of indol chromophores in Trp residues is sensitive to 


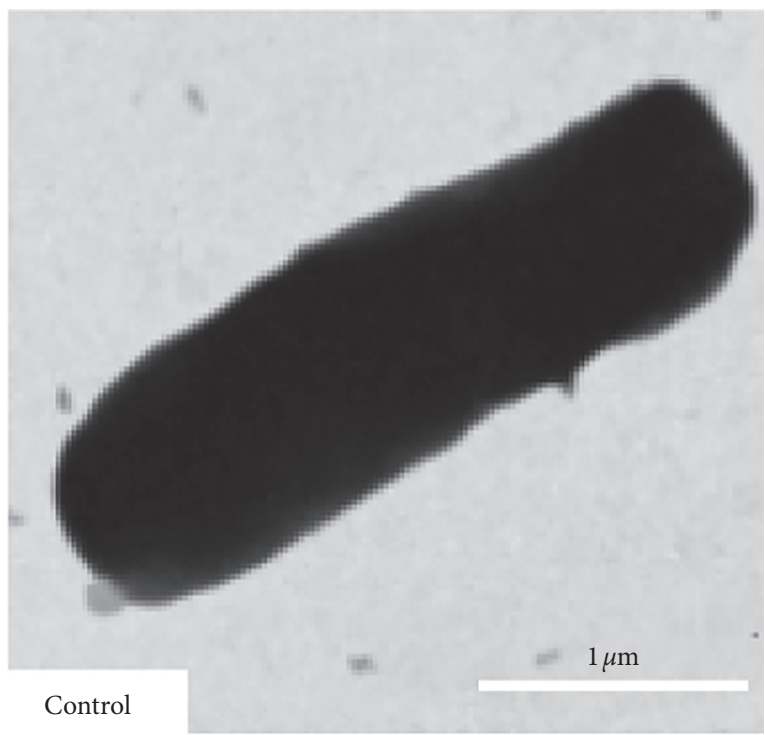

(a)

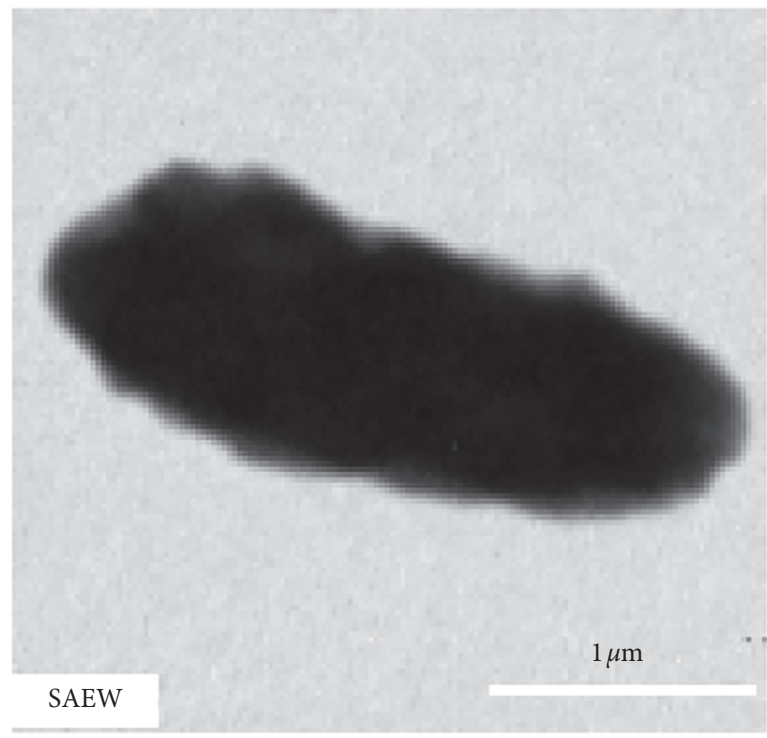

(c)

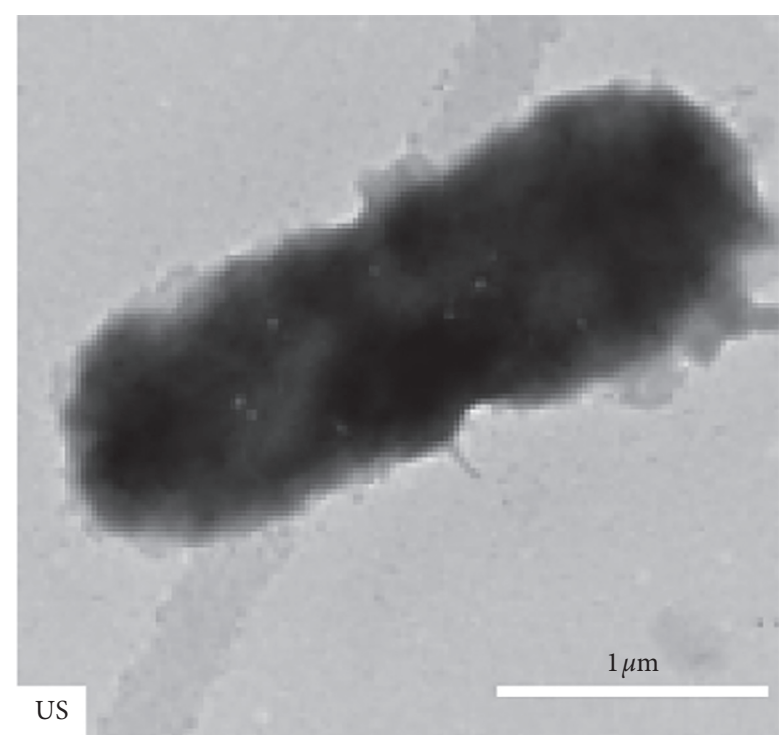

(b)

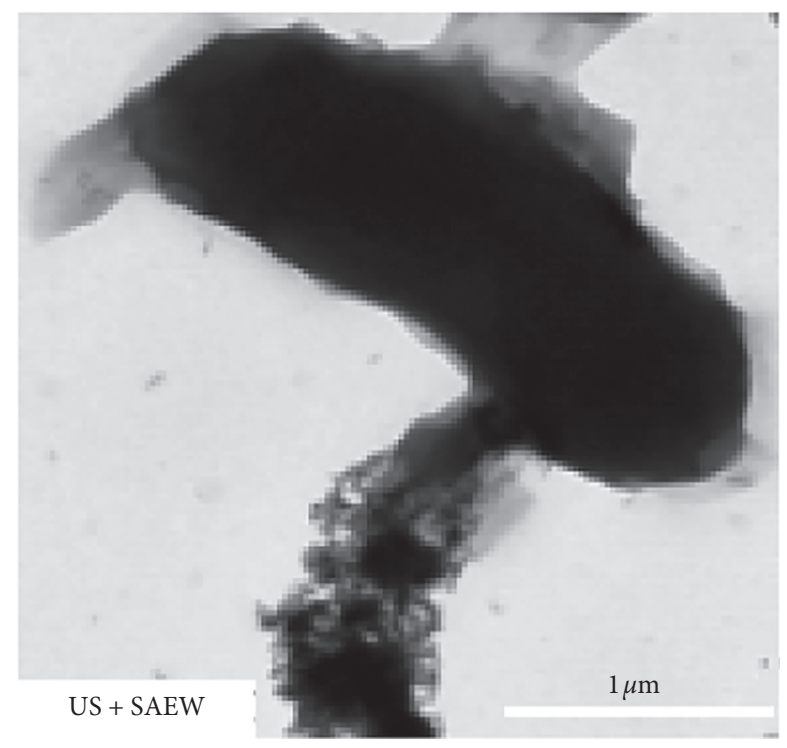

(d)

Figure 4: Transmission electron microscopy of E. coli after the single and combined treatment of ultrasound and slightly acidic electrolyzed water. Images were taken at magnification of $\times 5 \mathrm{~K}$. US: ultrasound, SAEW: slightly acidic electrolyzed water, and US + SAEW: ultrasound combined with slightly acidic electrolyzed water.

microenvironment particularities [27], which can provide information about the molecular microenvironment in the vicinity of the chromophores [28]. The spectrum of E. coli with different treatments is shown in Figure 6. Under the excitation wavelength of $278 \mathrm{~nm}$, the maximum emission wavelength of protein in control and US treated samples was $332 \mathrm{~nm}$. Nevertheless, the maximum emission wavelength of protein fluorescence was decreased after SAEW and US + SAEW treatments, which was $330 \mathrm{~nm}$, which suggested that SAEW could result in a blue shift of the maximum emission peak. In addition, it has been reported that US treatment could increase the fluorescence intensity of $E$. coli, thus improving the hydrophobicity of E. coli protein [29]. In the present study, the similar result was also observed after
US treatment. Nevertheless, SAEW and US + SAEW treatments reduced fluorescence intensity. The reduction of fluorescence intensity and the blue shift of the maximum emission peak implied the Trp residues transfer to a polar environment after SAEW and US + SAEW treatments [30].

The microenvironment of amino acid residues of biomolecules can be evaluated by synchronous fluorescence spectroscopy. The maximum emission wavelength of Tyr $(\lambda=15 \mathrm{~nm})$ was $283 \mathrm{~nm}$ under control and US treatments. However, it was decreased to $281.5 \mathrm{~nm}$ after SAEW and US + SAEW treatments (Figure 7(a)). The similar change of $\operatorname{Trp}(\lambda=60 \mathrm{~nm})$ was also observed. After SAEW and US + SAEW treatments, the maximum emission wavelength of Trp shifted from $278 \mathrm{~nm}$ to $276 \mathrm{~nm}$ 


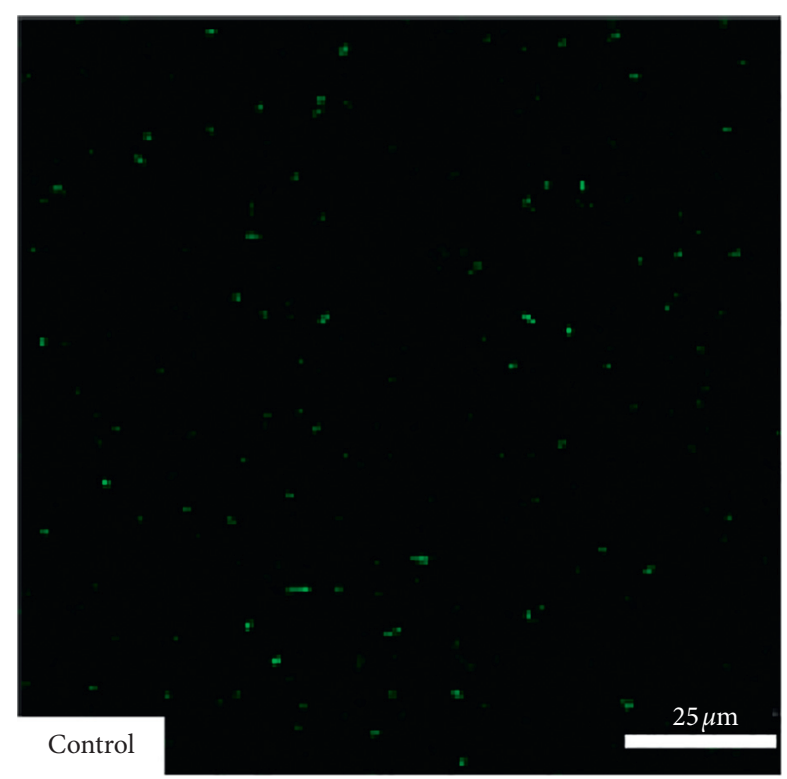

(a)

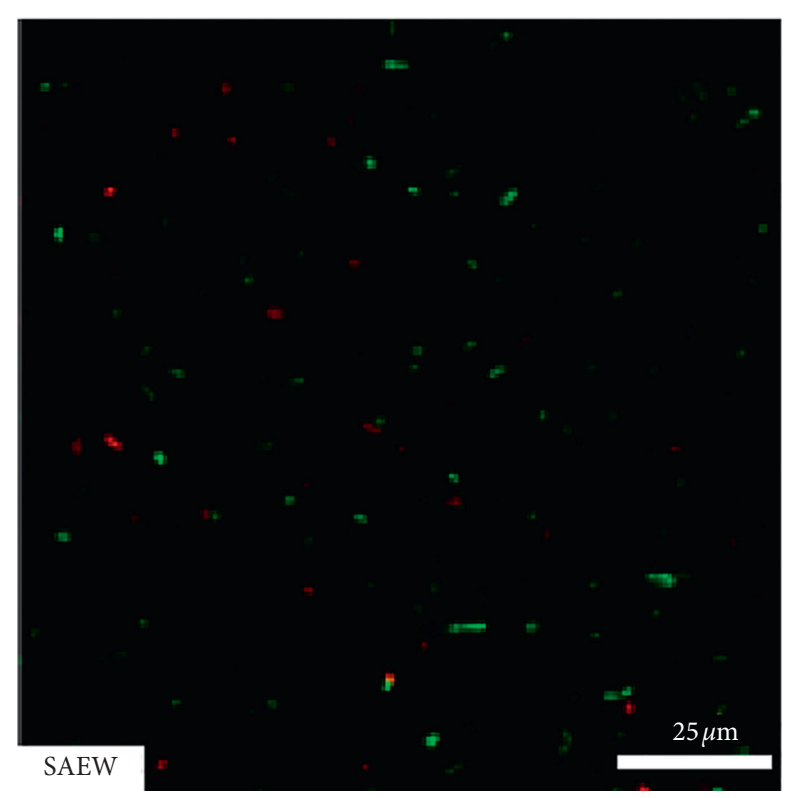

(c)

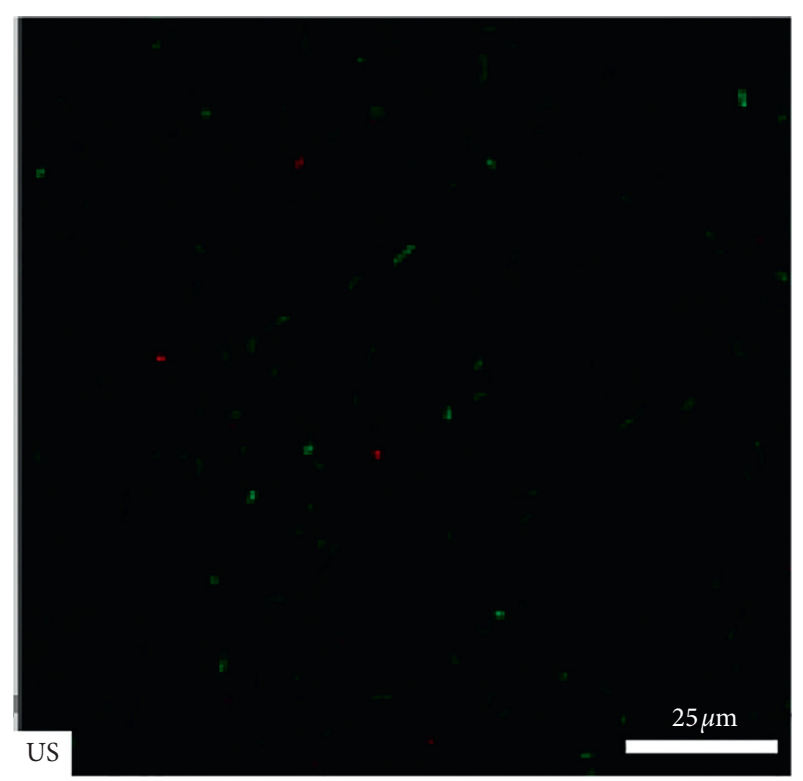

(b)

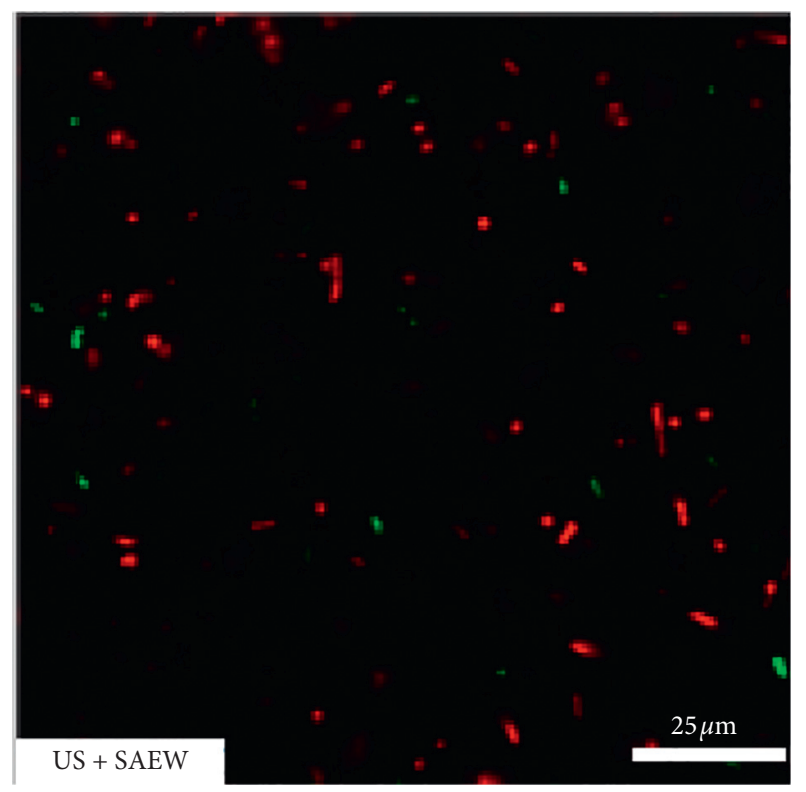

(d)

Figure 5: Confocal laser scanning micrographs of E. coli after the single and combined treatment of ultrasound and slightly acidic electrolyzed water. Images were taken at magnification of $\times 630$. US: ultrasound, SAEW: slightly acidic electrolyzed water, and US + SAEW: ultrasound plus slightly acidic electrolyzed water.

(Figure 7(b)). These results indicated that the polarity around the Tyr residues and Trp residues of E. coli decreased and the hydrophobicity increased after SAEW and US + SAEW treatment [27]. Additionally, the enhancement of fluorescence intensity was detected in US-treated E. coli, whereas the decrease of fluorescence intensity was found in
SAEW and US + SAEW treated samples. However, there was no significant difference between SAEW and US + SAEW treatment. The identical changes in resonance intensity of E. coli were also observed after US, SAEW, and US + SAEW treatments compared to the control (Figure 8). In the present study, US treatment enhanced the resonance 


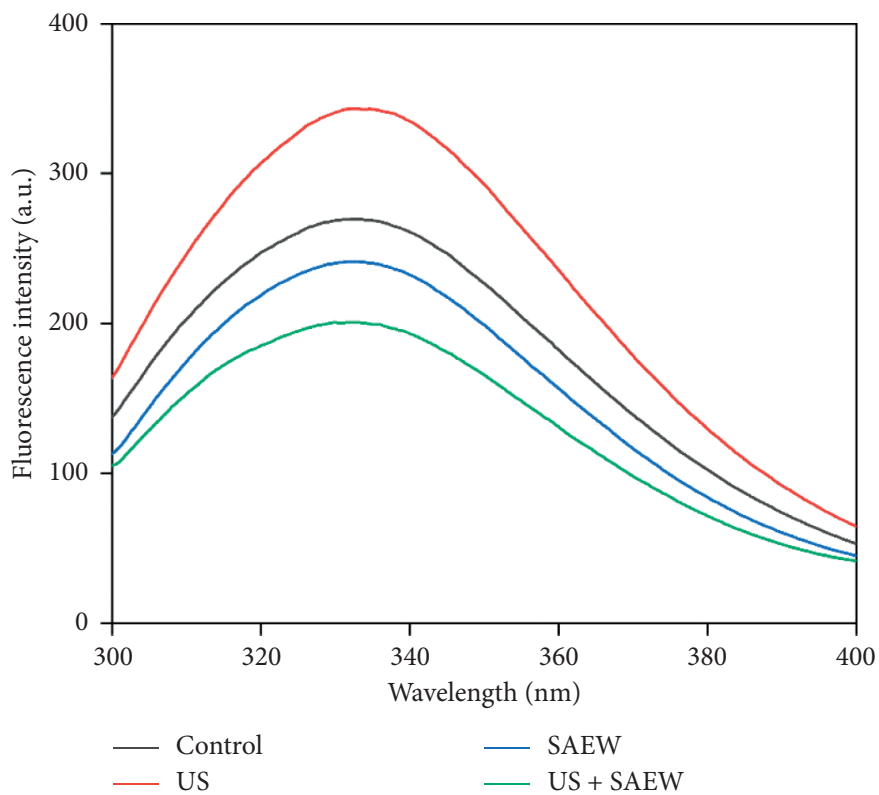

FIGURE 6: Endogenous fluorescence spectrometry of E. coli after the single and combined treatment of ultrasound and slightly acidic electrolyzed water. US: ultrasound, SAEW: slightly acidic electrolyzed water, and US + SAEW: ultrasound plus slightly acidic electrolyzed water.

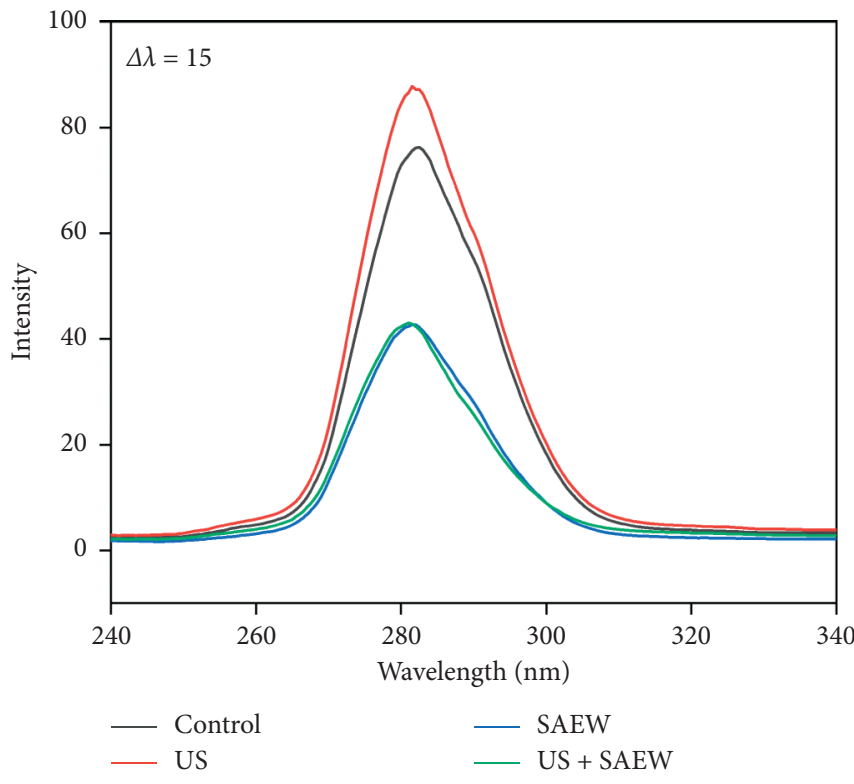

(a)

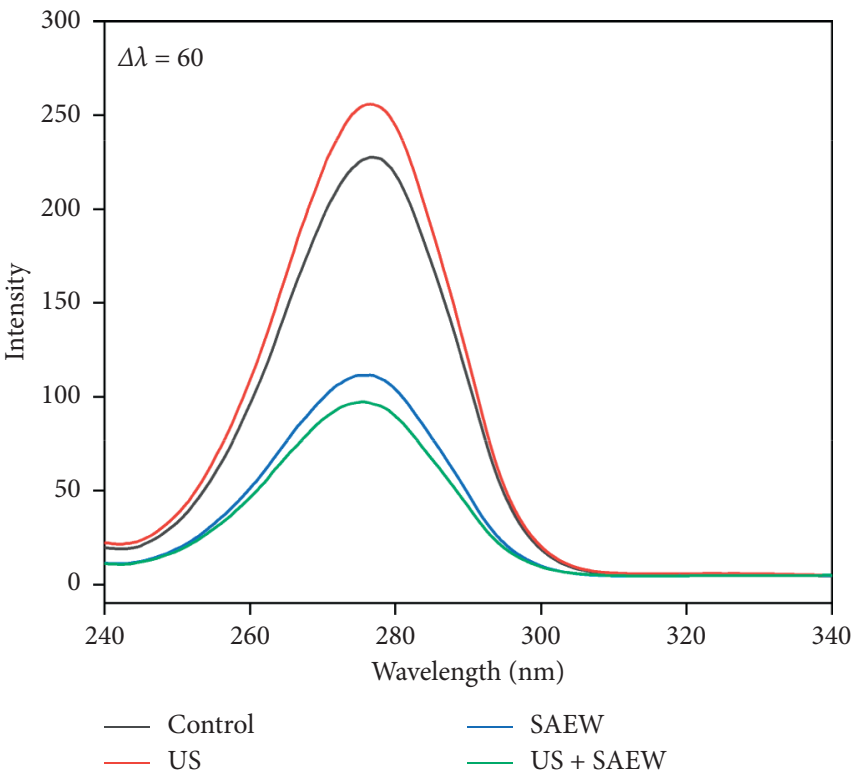

(b)

Figure 7: Synchronous fluorescence spectrometry of E. coli after the single and combined treatment of ultrasound and slightly acidic electrolyzed water. US: ultrasound, SAEW: slightly acidic electrolyzed water, and US + SAEW: ultrasound plus slightly acidic electrolyzed water. 


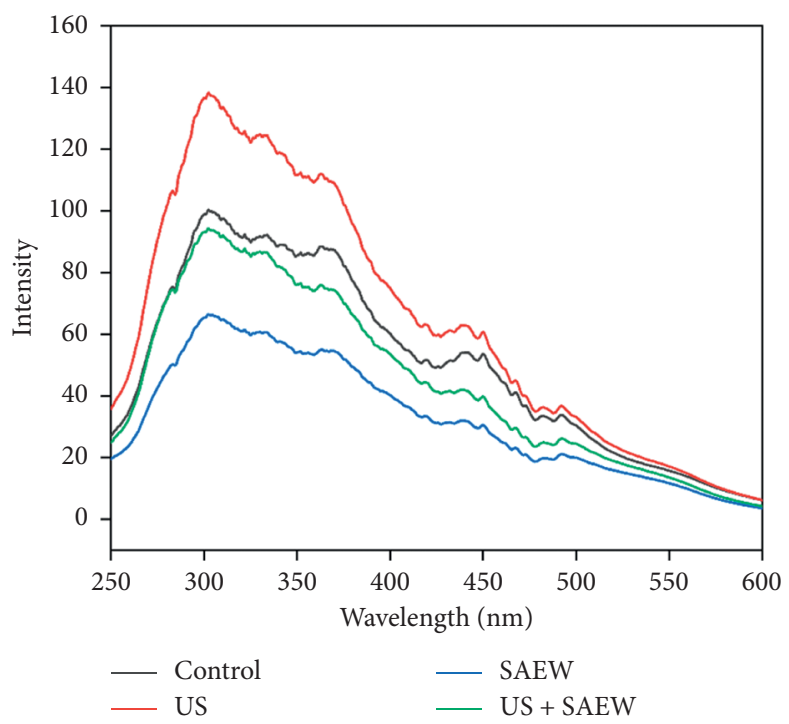

FIGURE 8: Resonance light scattering fluorescence spectrometry of E. coli after the single and combined treatment of ultrasound and slightly acidic electrolyzed water. US: ultrasound, SAEW: slightly acidic electrolyzed water, and US + SAEW: ultrasound plus slightly acidic electrolyzed water.

intensity of $E$. coli, indicating proteins of $E$. coli assembled. On the other hand, the decrease in resonance intensity of E. coli after SAEW treatment might be due to breakdown of protein [31].

\section{Conclusion}

US combined with SAEW showed the best sterilizing efficacy, with a significant reduction of survival cells compared with single US and SAEW treatments. Protein and potassium leakage tests as well as the morphologies of E. coli and CLSM analysis showed visible change under the combined treatment of US and SAEW. Fluorescence spectroscopy analysis found US and SAEW treatment changed membrane integrity and protein conformation of E. coli. In short, US treatment disrupted the cell membrane of E. coli and facilitated SAEW into the cells, thus improving the sterilizing effect. These results showed that US in combination with SAEW, as an environment friendly and safety sterilizing technology, could be developed as an effective and practical sterilizing method for food industry.

\section{Data Availability}

The data used to support the findings of this study are included within the article.

\section{Disclosure}

Xuecong Zhang and Liping Guo are the co-first authors.

\section{Conflicts of Interest}

The authors declare that there are no conflicts of interest.

\section{Authors' Contributions}

Liping Guo and Xuecong Zhang contributed equally to this work.

\section{Acknowledgments}

This work was supported by the financial support provided by the Shandong Modern Agricultural Technology and Industry System (SDAIT-11-11), China Agriculture Research System (CARS-41-Z06), and National Key R\&D Projects (2018YFD0501400).

\section{References}

[1] H. Cui, M. Bai, L. Yuan, D. Surendhiran, and L. Lin, "Sequential effect of phages and cold nitrogen plasma against Escherichia coli O157:H7 biofilms on different vegetables," International Journal of Food Microbiology, vol. 268, pp. 1-9, 2018.

[2] Q. Liu, L. Chen, A. K. C. Laserna, Y. He, X. Feng, and H. Yang, "Synergistic action of electrolyzed water and mild heat for enhanced microbial inactivation of Escherichia coli O157 : H7 revealed by metabolomics analysis," Food Control, vol. 110, Article ID 107026, 2020.

[3] A. A. Gabriel, "Combinations of selected physical and chemical hurdles to inactivate Escherichia coli O157: $\mathrm{H} 7$ in apple and orange juices," Food Control, vol. 50, pp. 722-728, 2015.

[4] A. Meireles, I. Machado, R. Fulgêncio, F. Mergulhão, L. Melo, and M. Simões, "Efficacy of antimicrobial combinations to reduce the use of sodium hypochlorite in the control of planktonic and sessile Escherichia coli," Biochemical Engineering Journal, vol. 104, pp. 115-122, 2015.

[5] J. Zhu, Y. Wang, X. Li et al., "Combined effect of ultrasound, heat, and pressure on Escherichia coli O157:H7, polyphenol oxidase activity, and anthocyanins in blueberry (Vaccinium 
corymbosum) juice," Ultrasonics Sonochemistry, vol. 37, pp. 251-259, 2017.

[6] S. Koda, M. Miyamoto, M. Toma, T. Matsuoka, and M. Maebayashi, "Inactivation of Escherichia coli and Streptococcus mutans by ultrasound at $500 \mathrm{kHz}$," Ultrasonics Sonochemistry, vol. 16, no. 5, pp. 655-659, 2009.

[7] D. Kang, Y. Jiang, L. Xing, G. Zhou, and W. Zhang, "Inactivation of Escherichia coli O157:H7 and Bacillus cereus by power ultrasound during the curing processing in brining liquid and beef," Food Research International, vol. 102, pp. 717-727, 2017.

[8] G. Sánchez, P. Elizaquível, R. Aznar, and M. V. Selma, "Virucidal effect of high power ultrasound combined with a chemical sanitizer containing peroxyacetic acid for water reconditioning in the fresh-cut industry," Food Control, vol. 52, pp. 126-131, 2015.

[9] L. Guo, Y. Sun, Y. Zhu et al., “The antibacterial mechanism of ultrasound in combination with sodium hypochlorite in the control of Escherichia coli," Food Research International, vol. 129, Article ID 108887, 2020.

[10] K. Luo, S. Y. Kim, J. Wang, and D.-H. Oh, "A combined hurdle approach of slightly acidic electrolyzed water simultaneous with ultrasound to inactivate Bacillus cereus on potato," LWT, vol. 73, pp. 615-621, 2016.

[11] S. M. E. Rahman, T. Ding, and D.-H. Oh, "Effectiveness of low concentration electrolyzed water to inactivate foodborne pathogens under different environmental conditions," International Journal of Food Microbiology, vol. 139, no. 3, pp. 147-153, 2010.

[12] A. Naka, M. Yakubo, K. Nakamura, and M. Kurahashini, "Effectiveness of slightly acidic electrolyzed water on bacteria reduction: in vitro and spray evaluation," Peer J, vol. 8, Article ID e8593, 2020.

[13] H. J. Kim, C. N. Tango, R. Chelliah, and D.-H. Oh, "Sanitization efficacy of slightly acidic electrolyzed water against pure cultures of Escherichia coli, Salmonella enterica, Typhimurium, Staphylococcus aureus and Bacillus cereus spores, in comparison with different water hardness," Scientific Reports, vol. 9, no. 1, p. 4348, 2019.

[14] A. J. Cichoski, D. R. M. Flores, C. R. De Menezes et al., "Ultrasound and slightly acid electrolyzed water application: an efficient combination to reduce the bacterial counts of chicken breast during pre-chilling," International Journal of Food Microbiology, vol. 301, pp. 27-33, 2019.

[15] J. Li, T. Ding, X. Liao, S. Chen, X. Ye, and D. Liu, "Synergetic effects of ultrasound and slightly acidic electrolyzed water against Staphylococcus aureus evaluated by flow cytometry and electron microscopy," Ultrasonics Sonochemistry, vol. 38, pp. 711-719, 2017.

[16] S. Gao, G. D. Lewis, M. Ashokkumar, and Y. Hemar, "Inactivation of microorganisms by low-frequency high-power ultrasound: 1. Effect of growth phase and capsule properties of the bacteria," Ultrasonics Sonochemistry, vol. 21, no. 1, pp. 446-453, 2014.

[17] M. M. Bradford, "A rapid and sensitive method for the quantitation of microgram quantities of protein utilizing the principle of protein-dye binding," Analytical Biochemistry, vol. 72, no. 1-2, pp. 248-254, 1976.

[18] C. Tang, B. Xie, and Z. Sun, "Antibacterial activity and mechanism of B-type oligomeric procyanidins from lotus seedpod on enterotoxigenic Escherichia coli," Journal of Functional Foods, vol. 38, pp. 454-463, 2017.

[19] S. Y. Park and S.-D. Ha, "Reduction of Escherichia coli and Vibrio parahaemolyticus counts on freshly sliced shad (konosirus punctatus) by combined treatment of slightly acidic electrolyzed water and ultrasound using response surface methodology," Food and Bioprocess Technology, vol. 8, no. 8, pp. 1762-1770, 2015.

[20] F. Forghani, M. Eskandari, and D.-H. Oh, "Application of slightly acidic electrolyzed water and ultrasound for microbial decontamination of kashk," Food Science and Biotechnology, vol. 24, no. 3, pp. 1011-1016, 2015.

[21] L. Zhao, Y. Zhang, and H. Yang, "Efficacy of low concentration neutralised electrolysed water and ultrasound combination for inactivating Escherichia coli ATCC 25922, Pichia pastoris GS115 and Aureobasidium pullulans 2012 on stainless steel coupons," Food Control, vol. 73, pp. 889-899, 2017.

[22] C. Ferreira, A. M. Pereira, M. C. Pereira, L. F. Melo, and M. Simoes, "Physiological changes induced by the quaternary ammonium compound benzyldimethyldodecylammonium chloride on Pseudomonas fluorescens," Journal of Antimicrobial Chemotherapy, vol. 66, no. 5, pp. 1036-1043, 2011.

[23] T. Ding, X.-T. Xuan, J. Li et al., "Disinfection efficacy and mechanism of slightly acidic electrolyzed water on Staphylococcus aureus in pure culture," Food Control, vol. 60, pp. 505-510, 2016.

[24] S. Nan, Y. Li, B. Wang, X. Cui, and W. Cao, "Effect of slightly acidic electrolyzed water for inactivating Escherichia coli O157:H7 and Staphylococcus aureus analyzed by transmission electron microscopy," Journal of Food Protection, vol. 73, no. 12, pp. 2211-2216, 2010.

[25] G. Liu, Z. Song, X. Yang, Y. Gao, C. Wang, and B. Sun, "Antibacterial mechanism of bifidocin A, a novel broadspectrum bacteriocin produced by Bifidobacterium animalis BB04," Food Control, vol. 62, pp. 309-316, 2016.

[26] M. E. Pacheco and L. Bruzzone, "Synchronous fluorescence spectrometry: conformational investigation or inner filter effect?" Journal of Luminescence, vol. 137, pp. 138-142, 2013.

[27] A. M. Simion, I. Aprodu, L. Dumitras, G. Elena Bahrim, P. Alexe, and S. Nicoleta, "Probing thermal stability of the $\beta$-lactoglobulin-oleic acid complex by fluorescence spectroscopy and molecular modeling," Journal of Molecular Structure, vol. 1095, pp. 26-33, 2015.

[28] M. Kong, X. G. Chen, C. S. Liu, C. G. Liu, X. H. Meng, and L. J. Yu, "Antibacterial mechanism of chitosan microspheres in a solid dispersing system against E. coli," Colloids and Surfaces B: Biointerfaces, vol. 65, no. 2, pp. 197-202, 2008.

[29] N. A. Aulik, D. N. Atapattu, C. J. Czuprynski, and D. R. McCaslin, "Brief heat treatment causes a structural change and enhances cytotoxicity of theEscherichia coli $\alpha$ hemolysin," Immunopharmacology and Immunotoxicology, vol. 35, no. 1, pp. 15-27, 2012.

[30] X. Zhao, Y. Li, H. Yuan, J. Yin, and M. Hu, "Antibacterial mechanism of octamethylene-1,8-bis (dodecyldimethylammonium bromide) against E. coli," Journal of Surfactants and Detergents, vol. 20, no. 3, pp. 717-723, 2017.

[31] A. Alimova, A. Katz, M. Siddique et al., "Native fluorescence changes induced by bactericidal agents," IEEE Sensors Journal, vol. 5, no. 4, pp. 704-711, 2005. 